\title{
VIDEOLAPAROSCOPIA NO TRAUMA ABDOMINAL
}

\section{LAPAROSCOPY IN ABDOMINAL TRAUMA}

\section{Sílvia Cristine Soldá, TCBC-SP ${ }^{1}$}

As possibilidades atuais para o diagnóstico de lesões no trauma abdominal abrangem um amplo arsenal de métodos invasivos e não invasivos e que podem ser usados de forma isolada ou em associação, tendo suas vantagens e desvantagens.

Esta diversidade permite uma investigação minuciosa, especialmente nos doentes estáveis, possibilitando critérios de seletividade na indicação de laparotomia. Desta maneira, pode-se estabelecer, com segurança, um algoritmo baseado nos recursos disponíveis, condições da instituição, levando em conta a relação custo/benefício e obviamente visando à melhor orientação para cada caso.

Dentre os inúmeros recursos diagnósticos na urgência, a laparoscopia tem representado uma contribuição significativa para a orientação terapêutica adequada.

Ela foi inicialmente realizada por Georg Kelling que, em 1901, apresentou no Congresso de Naturalistas e Médicos alemães um novo método endoscópico, o qual denominou celioscopia. Nesta época, utilizou um cistoscópio para visualizar a cavidade abdominal de cães.

No nosso meio, o pioneiro na sua utilização foi Azevedo, em 1945. Polak et al. contribuíram posteriormente com várias outras publicações.

Lamy \& Sarles, em 1956, foram os pioneiros no seu emprego na urgência traumática, ao avaliarem dois portadores de hemoperitônio por lesão do baço devida a trauma fechado. Desde então, a laparoscopia tem sido utilizada como recurso diagnóstico na urgência.

$\mathrm{Na}$ América Latina, particularmente com a divulgação de trabalhos cubanos e uruguaios, houve grande contribuição para sua maior aceitação, em função dos bons resultados.

Apesar da sua contribuição inegável no âmbito do diagnóstico de afecções abdominais eletivas há quase um século, seus horizontes ampliaram-se somente após o advento da videolaparoscopia que, empregada inicialmente no tratamento da colecistopatia calculosa, causou uma verdadeira revolução nos conceitos de técnica operatória.
A estabilidade hemodinâmica e a presença de uma equipe devidamente capacitada são condições fundamentais para a sua realização (Tabela 1).

Devido à visualização direta da cavidade abdominal, a laparoscopia pode diagnosticar lesões de forma segura através de sinais diretos e/ou indiretos.

Tabela 1

Condições para realização da laparoscopia na urgência

Estabilidade hemodinâmica

Sondagem nasogástrica e vesical

Monitoração cardíaca

Anestesia geral com controle de pressão endotraqueal

Equipe capacitada, com habilidade em procedimentos eletivos Aparelhagem e pinças adequadas para inventário

\section{Ferimentos por Arma Branca da Parede ANTERIOR do AbDOME}

Não se questiona a indicação cirúrgica sistemática para os doentes admitidos em choque, sinais de irritação peritoneal e evisceração.

O algoritmo preconizado para doentes assintomáticos consiste na exploração digital do ferimento que, quando positiva, indica a laparotomia exploradora. No entanto, observa-se que até $30 \%$ das intervenções são consideradas não terapêuticas, constatando-se apenas a penetração peritoneal.

Por outro lado, embora a simples observação ou realização de métodos de imagem sejam preconizadas por alguns autores, acreditamos que esta conduta não é segura pela limitação de tais métodos na identificação precoce de lesão de víscera oca.

1. Professora Assistente. Doutora do Serviço de Emergência do Departamento de Cirurgia da Faculdade de Ciências Médicas da Santa Casa de São Paulo

Recebido em 04/11/01

Aceito para publicação em 27/11/01

Trabalho realizado no Serviço de Emergência do Departamento de Cirurgia da Faculdade de Ciências Médicas da Santa Casa de São Paulo 
Mas, para os doentes assintomáticos, com exploração digital positiva para violação peritoneal, a videolaparoscopia possibilita um inventário seguro, pois as lesões de órgãos abdominais causadas por arma branca normalmente estão limitadas ao trajeto do agente agressor.

Além disso, é possível a identificação de sinais indiretos de lesão retroperitoneal, como a presença de hematoma, o que indica a conversão para a laparotomia, uma vez que há limitação no inventário da cavidade retroperitoneal.

A laparoscopia possibilita, portanto, o diagnóstico de penetração peritoneal, de lesão de órgão intracavitário e até mesmo o tratamento da lesão pela mesma via de acesso, em casos selecionados, com as vantagens de uma via de acesso menos invasiva que a laparotomia.

\section{Ferimentos por Arma Branca da Transição TÓRACO-ABDOMINAL}

Os ferimentos da transição tóraco-abdominal representam a principal causa de lesão do diafragma.

Quando existe lesão de víscera abdominal associada, os sinais de irritação peritoneal levam à indicação operatória e a lesão diafragmática acaba sendo um achado operatório.

No entanto, a lesão isolada do diafragma é caracterizada por uma série de dificuldades diagnósticas:

- sintomatologia frustra e exame físico normal em até $45 \%$ dos casos;

- radiografia de tórax normal em até $46 \%$ dos casos;

- exploração digital do ferimento, lavagem peritoneal, ultra-sonografia, tomografia com baixa especificidade. Isto talvez ocorra pelo pequeno tamanho: cerca de $70 \%$ dos ferimentos são menores que $2 \mathrm{~cm}$.

Existem outros recursos relatados para o diagnóstico da lesão diafragmática: realização de pneumoperitônio com o objetivo de provocar pneumotórax, introdução de contraste iodado na cavidade abdominal para que seja identificado no tórax, mas que também são falhos e caracterizados por um elevado índice de falsos negativos.

Muito embora as casuísticas relacionadas particularmente com as complicações de lesão por ferimento penetrante sejam raras, na verdade não se conhece a história natural de uma pequena lesão do diafragma que passe despercebida.

Feliciano et al., em 1998, analisaram 16 lesões do diafragma despercebidas, num período de nove anos e observaram que todas resultavam de ferimentos por arma branca ou por fogo. Em um doente, a radiografia de tórax foi normal; em nove não foi palpada solução de continuidade no diafragma durante a exploração digital do ferimento, realizada previamente à drenagem do tórax; em dois a lavagem peritoneal foi negativa e em quatro a lesão não foi diagnosticada durante a laparotomia.

Portanto, quando se preconiza a abordagem seletiva para os ferimentos dessa região, é necessário que seja adotado um método seguro, pois o diagnóstico tardio das hérnias diafragmáticas traumáticas, na vigência de complicações, revela índices de mortalidade de até $48 \%$.
Madden et al., em 1989, numa revisão de 28 publicações, observaram um índice de $20 \%$ de complicações de hérnias despercebidas, a maioria delas traduzida por estrangulamento intestinal, com um índice de 36\% de mortalidade.

A laparoscopia permite uma avaliação segura do diafragma, devido ao estiramento causado pelo pneumoperitônio, o que facilita a identificação de lesões, mesmo que pequenas.

Um fato interessante é que a lesão isolada do diafragma, que era observada em cerca de $2 \%$ a $12 \%$ dos ferimentos da transição tóraco-abdominal, passou a incidir em aproximadamente $25 \%$ dos casos após a utilização da videocirurgia, o que pode sugerir que alguns doentes submetidos a simples observação tenham recebido alta hospitalar com lesão despercebida.

Uma crítica que pode ser feita é o risco de pneumotórax durante a insuflação da cavidade abdominal com $\mathrm{CO}_{2}$.

Na nossa experiência essa complicação felizmente não foi observada, o que talvez possa ser explicado pelo gradiente de pressão existente entre as cavidades abdominal e torácica, que determina a sucção de vísceras abdominais para o tórax, "tamponando" o orifício diafragmático. Mesmo após a redução do epíplon para o abdome, não observamos esta ocorrência, possivelmente pela pressão positiva determinada pela ventilação mecânica.

Ivatury et al., em 1992, relataram um caso de pneumotórax hipertensivo numa série de doentes portadores de lesão do diafragma, e que necessitou de conversão para laparotomia.

Por este motivo, procuramos avaliar as repercussões hemodinâmicas e respiratórias da videolaparoscopia em modelo experimental de lesão do diafragma, utilizando suínos submetidos à monitoração cardíaca invasiva e não observamos alterações significativas no débito cardíaco, bem como de pressão de artéria pulmonar, quando utilizada pressão de pneumoperitônio de $16 \mathrm{mmHg}$.

Um outro aspecto que pode ser discutido é a possibilidade de aspiração de conteúdo gastrintestinal para o tórax quando existe lesão de víscera oca associada à lesão do diafragma, com risco de contaminação. Nesta eventualidade, é possível avaliar o tórax pela passagem da óptica através do orifício diafragmático, realizar a lavagem e aspiração do mesmo e até orientar a drenagem torácica.

A videotoracoscopia é um método que também pode ser utilizado nesta situação, o que tem sido motivo de controvérsia quanto à via de acesso mais adequada.

$\mathrm{Na}$ verdade, tanto a laparoscopia quanto a toracoscopia possibilitam o diagnóstico de maneira segura e a escolha normalmente está na dependência do método com o qual o cirurgião está mais familiarizado.

O fato é que, quando se realiza a toracoscopia e existe lesão do diafragma não é possível realizar o inventário da cavidade abdominal através da passagem da óptica pelo orifício, pois são necessárias pinças auxiliares para manipulação dos órgãos. Desta maneira, é mandatória a associação da laparoscopia (Tabela 2). 
Tabela 2

Toracoscopia x laparoscopia no diagnóstico da lesão do diafragma

\begin{tabular}{ll}
\hline Toracoscopia & Laparoscopia \\
\hline $\begin{array}{l}\text { Indicação: Raios X de tórax: hidrotórax } \\
\text { Derrame pleural }\end{array}$ & Radiografia normal \\
Avaliação adequada & Avaliação adequada \\
Intubação orotraqueal seletiva & $(-)$ \\
Lesão diafragma $\Rightarrow$ associar laparoscopia & Lesão diafragma $\Rightarrow$ sutura \\
avaliar tórax (mesma via)
\end{tabular}

Estamos convencidos do seu valor e acreditamos que sua indicação deva ser sistemática nos portadores de ferimentos penetrantes por arma branca da transição tóraco-abdominal assintomáticos, para diagnóstico de lesão do diafragma.

A escolha entre laparoscopia e toracoscopia estará na dependência da presença de derrame pleural à radiografia, situação na qual deve-se dar preferência à toracoscopia.

\section{Ferimentos por Arma de Fogo}

Para os ferimentos por arma de fogo a indicação cirúrgica é mandatória, em função do alto índice de lesões (cerca de $98 \%$ dos casos).

No entanto, nos ferimentos tangenciais da parede abdominal anterior, com orifícios de entrada e saída muito próximos e nos quais existe dúvida quanto à penetração na cavidade, o exame laparoscópico pode evitar a laparotomia desnecessária, caso não haja violação do peritônio.

Talvez essa seja a única indicação do exame sob anestesia local, pois o objetivo é visualizar o peritônio. Obviamente, diante da penetração, a laparotomia deve ser sistemática e imediata.

Sosa et al., em 1992, propuseram a utilização da laparoscopia nos ferimentos por arma de fogo na transição tóraco-abdominal à direita, em oito doentes nos quais havia dúvida quanto à penetração no abdome e descartaram penetração em seis deles. Pelo fato de que o fígado pode representar um "anteparo" ao projétil, a laparoscopia pode ser realizada de forma segura quando for constatada lesão única do diafragma ou até uma lesão hepática de pequeno tamanho e tangencial, sem sangramento ativo, embora esta seja uma indicação controversa.

\section{Trauma Abdominal Fechado}

No trauma abdominal fechado a laparoscopia parece não contribuir de maneira significativa, ao contrário do que se imaginava no inicio da experiência com o méto- do na urgência, pois não há vantagens sobre os métodos já consagrados, como a lavagem peritoneal, ultra-sonografia e tomografia, cuja utilização está na dependência de algoritmos bem definidos.

Porém, existem duas situações nas quais ela pode ser indicada:

- No doente submetido a tratamento não operatório de órgãos parenquimatosos (fígado, baço) e que evolui com dor abdominal deixando a dúvida quanto à possibilidade de lesão de víscera oca ou hemoperitônio.

A laparoscopia pode permitir uma orientação terapêutica correta, através da aspiração de hemoperitônio ou mesmo diagnóstico de lesão de víscera oca, lembrando que ao ser empregada, o tratamento deixa de ser não operatório, pois trata-se de um procedimento cirúrgico.

- Quando a tomografia revela a presença de líquido na cavidade abdominal e não constata a presença de lesão de víscera parenquimatosa que o justifique, a laparoscopia pode contribuir para o diagnóstico de lesão intestinal.

\section{Considerações Gerais}

$\mathrm{Na}$ análise da real contribuição da videolaparoscopia na urgência deve-se ressaltar que a avaliação da cavidade deve ser sistemática, através da visualização dos órgãos abdominais e do intestino em toda a sua extensão, usando-se pinças delicadas e apropriadas para "correr" as alças intestinais e permitir que o cirurgião realize uma "laparoscopia exploradora".

Além disso, devem ser considerados os riscos inerentes ao procedimento, como a possibilidade de lesões vasculares e de alças intestinais, além de complicações sistêmicas relacionadas ao pneumoperitônio, com repercussões hemodinâmicas e respiratórias, particularmente nos portadores de trauma cranioencefálico. Portanto, por 
ser invasivo, não é um procedimento isento de riscos e uma falha na sua indicação ou realização pode ser grave e até mesmo fatal.

Aliás, existem controvérsias quanto à possibilidade de complicações neurológicas no doente com trauma cranioencefálico submetido ao pneumoperitônio.

Rodrigues, em 1995, analisou os efeitos do pneumoperitônio com $\mathrm{CO}_{2}$ e hélio em modelo experimental de trauma cranioencefálico e observou aumento da pressão intracraniana independente do gás utilizado, o que traduz que a hipertensão seja decorrente do fator mecânico do pneumoperitônio.

Evidentemente, todos os procedimentos laparoscópicos devem estar apoiados na experiência, bom senso da equipe cirúrgica e em rigorosos critérios de seleção. Não se justifica o risco de, na tentativa de evitar laparotomias, aumentar o índice de complicações e mortalidade à cirurgia de urgência.

Diante da dúvida ou dificuldade técnica, impõe-se a laparotomia exploradora.

Por fim, é importante salientar que os algoritmos para o trauma abdominal devem estar baseados, sempre que possível, na análise dos mecanismos de trauma e que os métodos de imagem e invasivos podem ser utilizados de forma complementar, para uma avaliação diagnóstica segura.

\section{CONCLUSÕES}

Passados dez anos desde a primeira experiência com a videolaparoscopia no Serviço de Emergência da Faculdade de Ciências Médicas da Santa Casa de São Paulo, acreditamos que as indicações sistemáticas do método no trauma são:

- Ferimentos penetrantes por arma branca da transição tóraco-abdominal.

- Ferimentos tangenciais por arma de fogo da parede abdominal anterior.

Considerar em casos selecionados:

- Ferimentos por arma branca da parede abdominal anterior (exploração digital positiva, sem sinais abdominais).

- Dúvida quanto à possibilidade de lesão intestinal no doente internado para tratamento não operatório de lesão de órgão parenquimatoso.

- Ferimento por arma de fogo da transição tóraco-abdominal à direita, quando existe dúvida quanto a comprometimento abdominal.

\begin{abstract}
Despite the use of laparoscopy in abdominal trauma for several decades, it was only after the advent of video chip camera that an explosion of interest ocurred, giving rise to possibilities and perspectives not only in diagnosis but also in therapeutics. In trauma, its use has been gradually defined and experience has shown the benefits of the method in early diagnosis of visceral injuries as well as avoiding unnecessary laparotomies. Trauma laparoscopy is a safe method, can reduce negative and nontherapeutic laparotomies. The worrisome failure of laparoscopy to detect gastrintestinal injuries, specially small bowel lesions can be avoided with a mandatory and apropriated "run bowel" exploration. We believe that in hemodinamically stable patients, video laparoscopy is safely indicated in some situations in trauma, such as evaluation of diaphragmatic injuries in thoraco abdominal stab wounds and tangential gunshot wounds of anterior abdominal wall.
\end{abstract}

\title{
REFERÊNCIAS
}

1. Bard PA, Chen L. Subcutaneous emphysema associated with laparoscopy. Anaesth. Analg., 18: 48, 1969.

2. Berci G, Dunkelman D, Estephen LM et al. Emergency minilaparoscopy in abdominal trauma. Am. J. Surg., 146: 261, 1983.

3. Clay C, Hanlon CR. Pneumoperitoneum in the differential diagnosis of diaphragmatic hernia. J. Thorac. Surg., 20: $57,1951$.

4. Demetriades D, Kakoyiannis S, Parekh D et al. Penetrating injuries of the diaphragm. Br. J. Surg., 75: 971, 1988.

5. Fabian TC, Croce MA, Stewart RM et al. A prospective analysis of diagnostic laparoscopy in trauma. Ann. Surg., 217: 57, 1993
6. Fávero SSG, Maia Júnior AC, Munõz D et al. Ferimentos por projéteis de arma de fogo. Determinantes da lesão: revisão da literatura. Rev. Col. Bras. Cir., 5: 28795, 1995.

7. Feliciano DV, Cruse PA, Mattox KL et al. Delayed diagnosis of injuries to the diaphragm after penetrating wounds. J. Trauma, 28: 1135, 1988.

8. Frantzides CT, Carlson MA. Laparoscopic repair of a penetrating injury to the diaphragm : a case report. J. Laparoendosc. Surg., 4; 153,1994.

9. Freeman T7, Fischer RP. The inadequacy of peritoneal lavage in diagnosing acute diaphragmatic rupture. J. Trauma, 16: 538, 1976 
10. Ivatury R, Simon RJ, Weksler B et al. Laparoscopy in the evaluation of the intrathoracic abdomen after penetrating injury. J. Trauma - 33: 101, 1992.

11. Jacobaeus H, Apud Polak M. Kurze ubersicht uber Erfahringen dem Laparoskopie. Munch. Med. Wschr., 58: 217,1911

12. Kelling G, Apud Polak M. Veber die besichtigung der speidrrhore des magens mi biegsamen instrumenten. Verh. Ges. Detsch. Nauturfosch. Artze. II/2: 117, 1901.

13. Llanio R. Papel da laparoscopia propedêutica e terapêutica em afecções abdominais agudas. In: Castro L, Savassi Rocha PR, Carvalho DG. Tópicos em Gastroenterologia, 3, Belo Horizonte, 1992, p. 431-39.

14. Llanio R, Sotto A, Jimenez G. et al. Resultados obtenidos con la laparoscopia de urgência. Reporte de 1509 casos. Rev. Cub. Cir., 12: 23, 1973.

15. Livingston DH, Bartholomeu JT, Blackwood J et al. The role of laparoscopy in abdominal trauma. J. Trauma, 33: 471, 1992.

16. Madden MR, Paull DE, Finkelstein JL. Occult diaphragmatic injury from stab wounds to the lower chest and abdomen. J. Trauma, 29: 292, 1989.

17. Maffa SM, Quinn JV, Slotman GJ. Hemodynamic effects of carbon dioxide pneumoperitoneum during mechanical ventilation and positive end-expiratory pressure. J Trauma, 35: 613, 1993.

18. Merlotti GJ, Marcel G, Sheaff CM et al. Use of peritoneal lavage to evaluate abdominal penetration. J. Trauma, 25: 228, 1985.

19. Moore JB, Thompson D. Abdominal injuries associated with penetrating trauma in the lower chest. Am. J. Surg., 140: 724, 1980 .

20. Negrotto G, Zeballo E, Turturiello H et al. La laparoscopia en la urgência. Arch. Med. Int. (Uruguay), 5: 55-66, 1983.

21. Osler TM. The role of laparoscopy in abdominal trauma: A technic in search of an indication? Int. Surg., 79: 304, 1994.

22. Polak M, Montenegro MR, Meira JA et al. Schistosomiasis Mansoni: Pathological changes in the liver in different stages of the disease by means of laparoscopy and needle biopsy. Rev. Inst. Med. Trop. S. Paulo. 1: $18,1959$.

23. Polak M. Laparoscopia, São Paulo, Sarvier, 1957, 139p.

24. Rasslan S, Rodrigues FCM, Soldá SC. Análise crítica da videolaparoscopia na urgência abdominal não traumática. In: Margarido NF, Saad Jr. R, Cecconello I, Martins JL, de Paula RA, Soares RLA. Vídeocirurgia. Robe, Ed.: São Paulo, p.278, 1993.
25. Rasslan S, Rodrigues FCM, Soldá SC. Vídeo-laparoscopia diagnóstica e terapêutica na urgência. Arq. Bras. Cir. Dig. 121, 1993.

26. Rodrigues FCM. Efeitos do pneumoperitônio com gás carbônico e hélio na pressão intracraniana e na pressão de perfusão cerebral em modelo experimental de trauma crânio-encefálico. Tese de Doutorado. Faculdade de Ciências Médicas da Santa Casa de São Paulo. São Paulo, 1995.

27. Root HD, Hauser CW, McKinley et al. Diagnostic peritoneal lavage. Surg., 57: 633, 1965.

28. Sackier JM. Laparoscopy in the emergency setting. World. J. Surg., 16: 1084, 1992.

29. Salvino CK, Esposito TJ, Marshall WJ. et al. The role of diagnostic laparoscopy in the management of trauma patients: a preliminary assessment. J. Trauma, 34: $506,1993$.

30. Soldá SC. Laparoscopia diagnóstica no trauma abdominal. Dissertação de Mestrado. Faculdade de Ciências Médicas da Santa Casa de São Paulo, São Paulo, 1993.

31. Sosa JL, Puente MD. Laparoscopy in the evaluation and management of abdominal trauma. Int. Surg., 79: 307, 1994.

32. Thompson JS, Moore EE. Peritoneal lavage in the evaluation of penetrating abdominal trauma. Surg. Gynecol. Obstet., 153: 861, 1981.

33. Towsend MC, Slanceum L, Chobaw PS et al. Diagnostic laparoscopy as an adjunt to seletive conservative management of solid injuries after blunt abdominal trauma. J. Trauma, 35: 647, 1993.

34. Westerband A, Water JM, Amzallag M et al. Cardiovascular changes during laparoscopic cholecystectomy. Surg. Gynecol. Obstet., 175: 535, 1992.

Endereço para correspondência:

Dra. Sílvia Cristine Soldá

Av. Jacutinga, 493/81

04515-030 — São Paulo-SP 